\title{
CARACTERÍSTICAS FÍSICO-QUÍMICAS DE LAS AGUAS SUPERFICIALES DE LA RAMBLA DEL MORO DESPUÉS DE UNA RIADA (CUENCA DEL RÍO SEGURA, SE DE ESPAÑA)
}

\author{
M. Ortega, M.R. Vidal-Abarca, M. Suárez, J.L. González-Beseran y L. Ramírez-Díaz \\ Departamento de Biología Animal y Ecología, Facultad de Biologia, Universidad de Murcia. España.
}

Palabras clave: «Rambla»,flash flood, physico-chemical characteristics, Segura River.

\begin{abstract}
PHYSICO-CHEMICAL CHARACTERISTICS IN THE RAMBLA DEL MORO POSTFLOOD (RIVER SEGURA WATERSHED; S.E. OF SPAIN)

Changes in the physico-chemical parameters induced by a flash flood (7 October 1986) in the Rambla del Moro (River Segura watershed), are studied analyzing the water along ten days after the flood. Suspended solids, alkalinity and nutrients (nitrate-nitrogen and phosphte-phosphorus), increase markedly after the flood while conductivity decline quickly in relation with the peaks of discharge. Oxygen concentation doesn't change during the study. Correlation between the physico-chemical parameters and the discharge are discussed.
\end{abstract}

\section{INTRODUCCIÓN}

Las riadas o avenidas de agua, constituyen uno de los fenómenos hidrológicos naturales más característicos de las cuencas mediterráneas (SundBorG, 1983). Ligadas a distintos factores ambientales (climáticos, del sustrato, del relieve, etc.) de las cuencas de drenaje y a la ausencia de una cobertura vegetal importante (LULL \& REINHART, 1972), se han descrito como sucesos catastróficos y perturbadores de la dinámica fluvial, con efectos sobre la composición físicoquímica del agua (Fischer \& MinCKLEY, 1978), los organismos acuáticos (FISCHER et al., 1982) y sobre la cantidad de materiales que transfieren, en la cuenca, desde la cabecera a la desembocadura (BORMANN \& LiKENS, 1979).

Aunque en España es fácil encontrar una ámplia literatura sobre las avenidas de agua y sus efectos devastadores sobre la población humana

Limnética, 4: 19-26 (1988)

(C) Asociación Española de Limnología, Madrid, Spain
(M.O.P.U., en prensa), existe un total desconocimiento de los cambios que provocan en la composición físico-química del agua, en la estructura y funcionamiento de las comunidades acuáticas $\mathrm{y}$, en definitiva, en la dinámica fluvial. Únicamente se encuentran referencias aisladas en Sabater (1981); Ávila (1984); Escarré et al. (1984); VidAl-AbarCa (1985); SuÁrez (1986) y PUIG et al. (1987), sobre los cambios que experimenta algún parámetro físico-químico, en concreto, o el lavado y arrastre de poblaciones de algas y macroinvertebrados.

En la cuenca del río Segura, las riadas se suceden con cierta frecuencia (LÓPEz BERMúdez et al., 1979) y los fenómenos climáticos que desencadenan las lluvias torrenciales en esta región han sido descritos por LóPEZ BERMúDEZ \& GUTIÉRREZ (1983).

En este trabajo, se describe la evolución de diferentes parámetros físicos y químicos, después de producirse una importante riada en la Rambla del Moro, así como sus relaciones con el caudal de agua. 


\section{MATERIAL Y MÉTODOS}

\section{Área de estudio}

La Rambla del Moro, localizada en el NE de la cuenca del río Segura (Figura la), es uno de sus afluentes por la márgen izquierda, más irregulares en relación al caudal de agua que aportan.

En la Tabla 1, se presentan algunas de las características morfométricas más relevantes de esta subcuenca. Se trata de una red de drenaje muy densa y altamente jerarquizada. Su perfil longitudinal muestra una evolución equilibrada con pendiente muy acusada en cabecera (Figura 1d). El $90 \%$ de los cauces de su cuenca se comportan como ramblas, habitualmente secas (sólo transportan agua, de evacuación muy rápi- da, cuando se producen fuertes precipitaciones). El resto mantiene agua de forma permanente o temporal pero el tiempo suficiente, en este último caso, como para que se desarrolle una comunidad estable de organismos acuáticos (Hynes, 1970).

La cuenca de la Rambla del Moro se asienta sobre terrenos calizos y margo-arcillosos, muy blandos y fácilmente erosionables. La cubierta vegetal, muy degradada debido a factores antrópicos, está constituida por especies nitrófilas; en las zonas bajas por tomillares muy aclarados y en las más altas por bosquetes aislados de Pinus halepensis. El clima es seco y cálido, de relativa aridez, con precipitaciones medias anuales inferiores a $300 \mathrm{~mm}$ y temperaturas medias anuales que oscilan entre 16 y 18 " C (VIDAL-AbARCA et al. en prensa). Cabe destacar que el máximo pluvio-

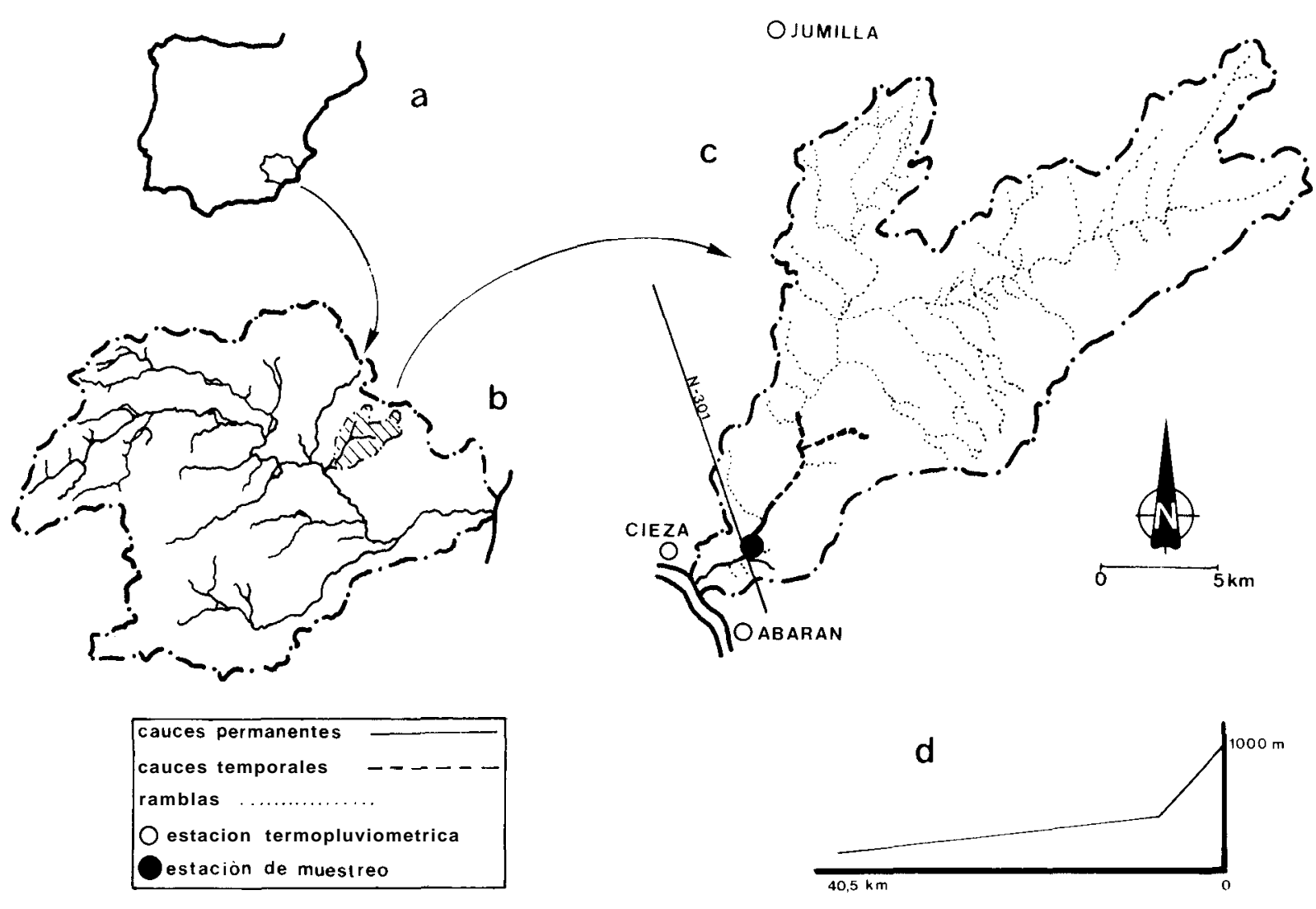

Figura 1.- Situación geográfica del área de estudio $(a, b)$, localización de las estaciones termopluviométricas y punto de muestreo (c), y perfil longitudinal (d) de la Rambla del Moro.

Study area (a,b), thermopluviometrical stations and sampling site (c), and longitudinal profil (d) of the Rambla of Moro. 
Tabla 1.- Características morfométricas más importantes, de la Cuenca de la Rambla del Moro Main morphometrical characteristics of thc Rambla del Moro.

\begin{tabular}{ccccc}
$\begin{array}{c}\text { Superficie total } \\
\text { de la cuenca } \\
\mathrm{Km}^{2}\end{array}$ & $\begin{array}{c}\text { Altitud } \\
\text { media } \\
\mathrm{m}\end{array}$ & $\begin{array}{c}\text { Longitud total } \\
\text { del cauce } \\
\mathrm{Km}\end{array}$ & $\begin{array}{c}\text { Pendiente } \\
0 / 00\end{array}$ & $\begin{array}{c}\% \text { Cauces } \\
\text { con agua }\end{array}$ \\
\hline 390,64 & 580 & 40,5 & 20,74 & 10 \\
\hline
\end{tabular}

métrico anual se produce preferentemente en otoño.

La zona donde se realizó el estudio se encuentra en el cruce de la rambla con la Carretera Nacional 301 (Figura 1c). La anchura del cauce oscilaba entre 1,5 y $3,6 \mathrm{~m}$ y la profundidad de la lámina de agua entre 5 y $60 \mathrm{~cm}$, según el caudal circulante. El lecho del cauce estaba formado por grava y arena embebidas en una matriz arcillosa, con un tapiz vegetal acuático muy denso en las zonas deposicionales, constituido por Chara vulgaris var. crassicaulis, Zannichellia palustris, Cladophora glomerata, Spirogyra sp. y Enteromorpha sp. La vegetación de ribera estaba compuesta por Juncus sp. Carex sp.; Phragmites sp.; Tamarix gallica; etc.

\section{Métodos de muestreo y análisis}

Se tomaron 10 muestras a lo largo de $10 \mathrm{~m}$ de cauce, en días consecutivos ( 9 al 18 de octubre de 1986) después de producirse la riada el día 7 , una vez se pudo acceder a la rambla. Durante este período la temperatura media del agua fue de $20,8^{\circ} \mathrm{C}$ y la velocidad de la corriente osciló entre 20 y $75 \mathrm{cmls}$, siendo su valor medio de $50,1 \mathrm{cmls}$. Una vez estabilizado el caudal, se realizó otro muestreo el día 3 de noviembre, para poder constatar los resultados obtenidos.

Los datos de precipitación diaria analizados, facilitados por el Centro Meteorológico Zonal de Murcia, corresponden a las estaciones meteorológicas de Abarán, Jumilla y Cieza (Figura 1c), al no existir una en la zona de estudio.

La ausencia de una estación de aforo en la Rambla del Moro impidió conocer los módulos absolutos durante estos días, así como los registrados durante la riada. Se han calculado módulos relativos mediante la fórmula: Velocidad instan- tánea de la corriente $\mathrm{x}$ Anchura $\mathrm{x}$ Profundidad de la lámina de agua (MARGALEF, 1983).

Los métodos de toma de muestras y análisis de los parámetros físicos y químicos medidos (tabla 3) se describen en Golterman et al. (1978), INSTITUTO DE HIDROLOGía (1980) y RODIER (1981), entre otras publicaciones.

\section{RESULTADOS}

\section{Característicashidrológicas (Tabla 3)}

Las precipitaciones de los días $3,4,5$ y 6 provocaron la riada del día 7 , según se observa en los histogramas de precipitación (Figura 2A).

En la figura $2 \mathrm{~B}$ se presentan los valores de caudal, medidos durante los días de muestreo, siendo la evolución del módulo relativo paralela a las variaciones de la precipitación (fig. 2A). Las lluvias de los días 12 y 16 de octubre provocaron dos nuevos picos acusados de caudal los días 13 y 17-18, respectivamente. Este hecho dificulta la interpretación de los resultados, dado que se interrumpe el proceso de estabilización físicoquímica del agua.

Los valores de caudal y velocidad instantánea de la corriente más elevados se registraron el $9^{\circ}$ día de muestreo, coincidiendo con el 2" pico de precipitación, que, aunque no fué el más alto se debió a una lluvia muy localizada en el punto de muestreo.

Los valores de caudal y de velocidad instantánea de la corriente medidos el día 3 de noviembre, son muy próximos a las condiciones anteriores al inicio del estudio, con lo que se puede asegurar la estabilización, al menos de las condiciones fisionómicas de la rambla después de producirse la riada. 

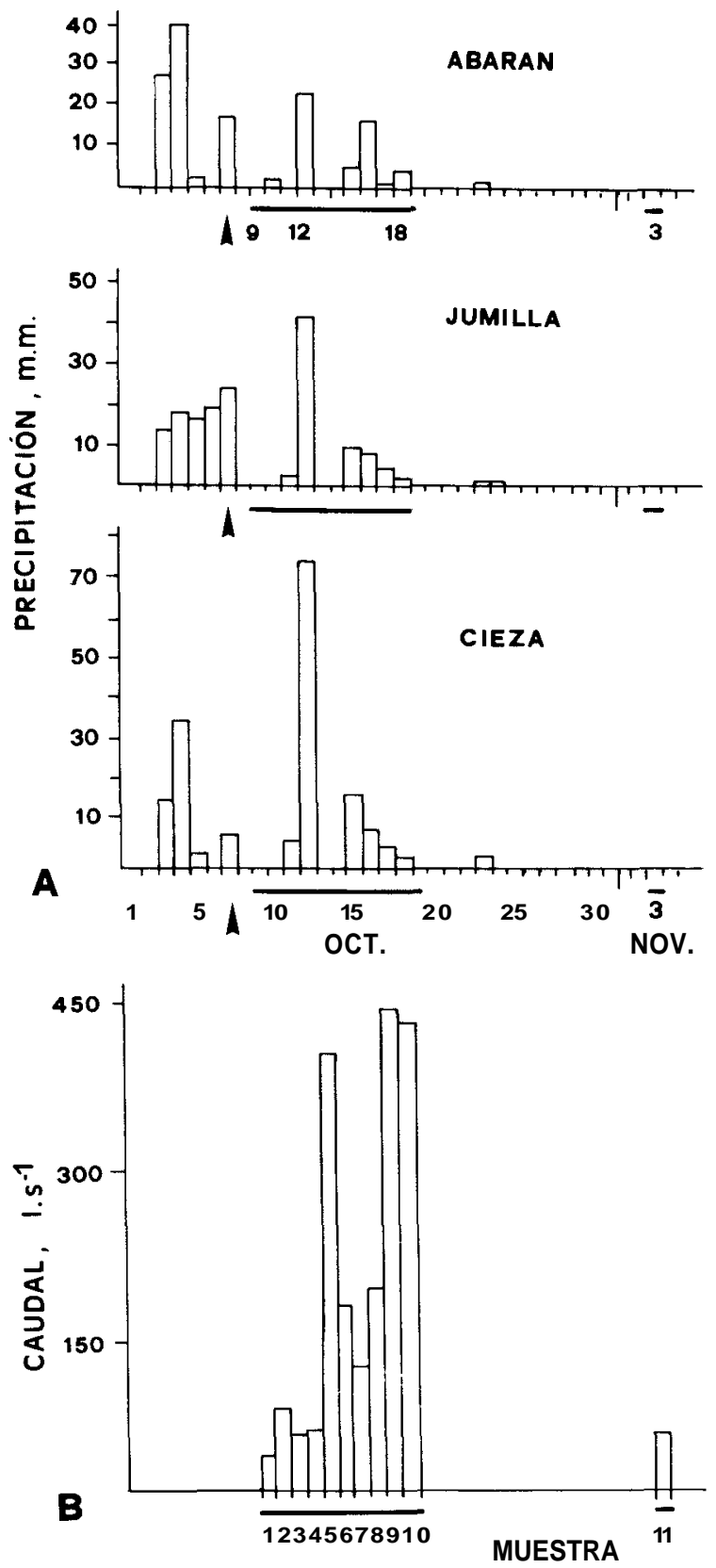

Figura 2.- A-Histogramas de la precipitación diaria de octubre y principios de noviembre de 1986, en tres estaciones termopluviométricas cercanas a la Rambla del Moro (Abarán, Jumilla y Cieza). Se señala con punta de flecha el día de la nada. B-Evolución del caudal de agua (l/s), durante los días del estudio, después de producirse la nada en la Rambla del Moro.

A-Daily rainfall during October-November 1986 in 3 thermopluviometrical stations located near the Rambla del Moro (Abarán, Jumilla and Cieza). Arrow indicate the flash flood. B-Discharge after the flood on the Rambla del Moro.

\section{Características físico-químicas (Tabla 3)}

Para poder contrastar los resultados obtenidos se presentan las características físico-químicas de las aguas superficiales de la rambla en dos fases del ciclo hidrológico de 1982-83 (otoño e invierno), en condiciones normales (Tabla 2). En general, se trata de aguas alcalinas, tamponadas y muy mineralizadas, debido fundamentalmente a los cloruros provenientes del tipo de sustrato que drena la red fluvial (margas del Keuper) (VIDALABARCA, 198.5). Las concentraciones de nitrógeno y fósforo, relativamente elevadas, corresponden a aguas eutrofizadas.

En la Figura 3, se presenta la evolución de los parámetros físicos y químicos medidos. En relación con los sólidos en suspensión (Figura 3A), y teniendo en cuenta los valores de este parámetro medido en condiciones normales (Tabla 2), su incremento en el agua de la rambla llega a ser de hasta un $2.000 \%$.

La conductividad (Figura 3A) acusa una fuerte disminución con el aumento de caudal debido posiblemente a fenómenos de dilución (FIsCHER \& MinCKLEY, 1978), correspondiéndose los picos de caudal con los valores mínimos de conductividad.

La variación inversa de la alcalinidad y el $\mathrm{pH}$ se presenta en la figura $3 \mathrm{~B}$. El $\mathrm{pH}$ responde al primer pico de caudal con una brusca disminución, siendo 6,3 el valor mínimo detectado durante el estudio. La alcalinidad, por el contrario, sufre un ligero aumento, aunque el valor máximo (2,52 meq/1) se registró el 10" día de muestreo después de producirse el 2 " pico de caudal. Sin embargo, las variaciones en ambos parámetros son mínimas (ver Tabla 2) como corresponde a aguas extraordinariamente tamponadas. Parece, pues, que el aumento de caudal no desequilibra el sistema tampón de las aguas de la rambla.

Los nutrientes (nitratos y fosfatos, Figura 3C) experimentan de forma absoluta un importante aumento, alrededor de un $300 \%$, en ambos parámetros. Aunque los nitratos no responden de forma inmediata al primer pico de caudal, se produce un aumento brusco en el $2^{\circ}$, que se mantiene durante más tiempo que en el caso de los fosfatos, cuya concentración el último día de muestreo, fué de 0,76 yg-at ${\mathrm{P} . \mathrm{PO}^{3-}}_{4} / \mathrm{l}$, similar a 
las condiciones habituales de la rambla.

Las variaciones en la concentración de oxígeno disuelto son mínimas y no responden de forma general a los pulsos de caudal. Hay'que tener en cuenta que durante todo el estudio hubo sobresaturación de oxígeno disuelto en el agua.

\section{DISCUSIÓN}

A menudo, las riadas se han interpretado como un fenómeno de dilución de la carga contaminante de un río o del grado de salinidad del agua (Fischer \& Minckley, 1978). Sin embargo, esta visión resulta extraordinariamente simplista a juzgar por los intensos cambios que sufre la composición físico-química de las aguas de la Rambla del Moro después de la riada de octubre de 1986. A excepción del oxígeno disuelto, el resto de los parámetros físico-químicos medidos experimentan fuertes oscilaciones en relación con el caudal.

Los sólidos en suspensión proporcionan el coeficiente de correlación más elevado con el caudal y altamente significativo $(r=0,92$; $\mathrm{p} \leqslant 0,01$ ), como corresponde a una cuenca desforestada, de sustrato blando y fácilmente erosionable. No cabe duda que uno de los efectos más drásticos de las avenidas de agua consiste en la transferencia de suelo desde las partes más altas de la cuenca hasta la desembocadura (SUNDBORG, 1983).

Entre la conductividad y el caudal existe una correlación inversa y significativa $(\mathrm{r}=-\mathbf{0 , 8}$; $\mathrm{p} \leqslant 0,01$ ). FISCHER \& MinCKLEY (1978) explican este efecto por dilución. Los valores bajos de conductividad del agua de lluvia diluirían las concentraciones de los aniones presentes en el agua que circula por los cauces, en equilibrio químico con el sustrato $\mathrm{y}$, por tanto, muy ricas en sales disueltas. Sin embargo, Fischer et al. (1982), analizando los efectos posteriores a una avenida de agua en un río del desierto de Arizona, encuentran que la conductividad, los sulfatos, los cloruros y la alcalinidad son extraordinariamente constantes a lo largo del estudio. EsCARRÉ et al. (1984), estableciendo el balance de nutrientes en una cuenca de encinar en el Pradés, concluyen que el sistema exporta a través del
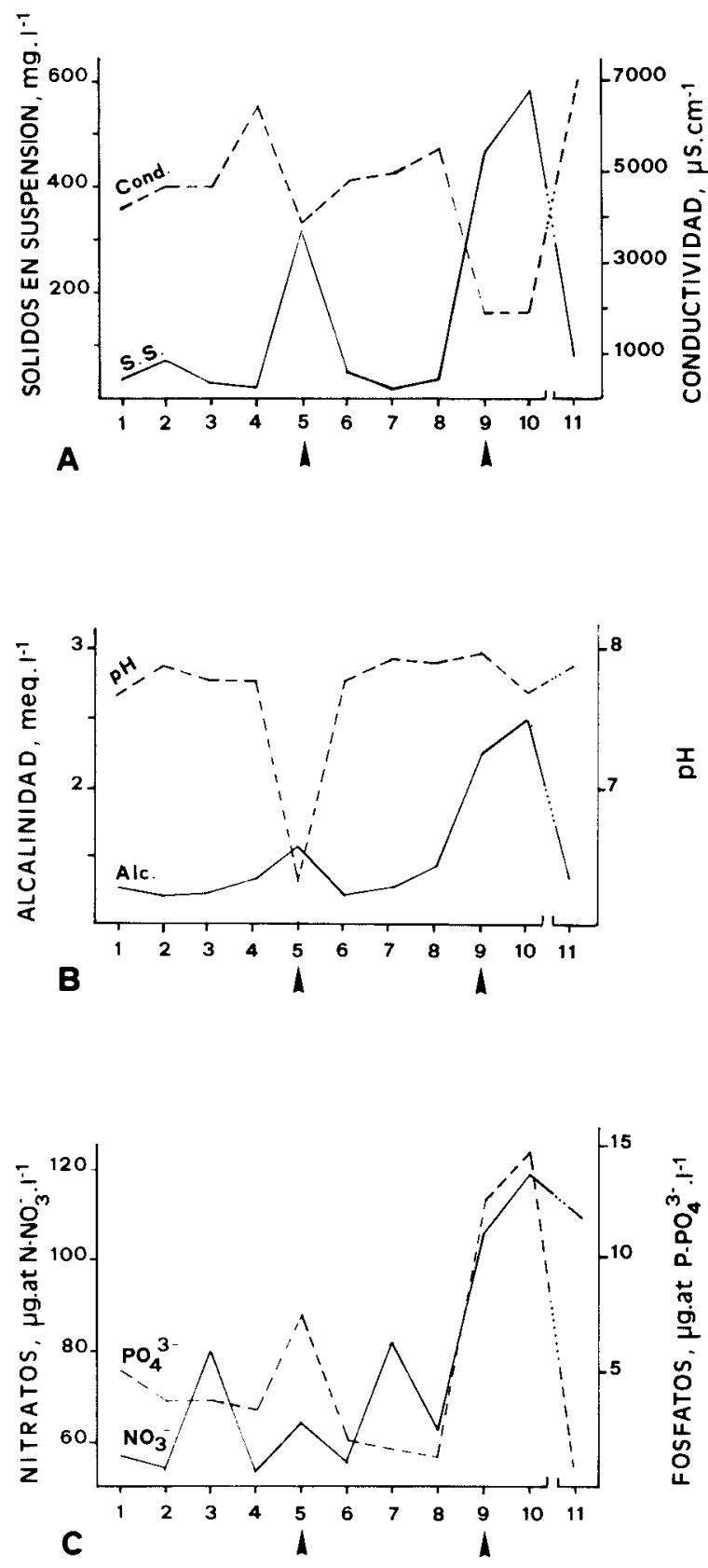

Figura 3.- Evolución de los sólidos en suspensión y de la conductividad (A), alcalinidad y $\mathrm{pH}$ (B), nitratos y fosfatos (C), durante los días del estudio, después de producirse la riada en la Rambla del Moro. Se señalan con punta de flecha los días de máximo caudal.

Suspended solids and conductivity (A), alkalinity and pH (B), disolved nitrate-nitrogen and phosphate-phosphorus (C), after the flood in the Rambla del Moro. Arrows denote time of mayor flood peaks. 
Tabla 2.- Características físicas y químicas de las aguas superficiales de la Rambla del Moro, en dos fases del ciclo hidrólogico 1982-83 (otoño e invierno), en condiciones normales.

Physico-chemical characteristics of the Rambla del Moro during autumn and winter of the hydrological cycle $1982-83$.

\begin{tabular}{|c|c|c|}
\hline Parámetros fisico-químicos & $\begin{array}{c}\text { Otoño } \\
\text { 3-11-82 }\end{array}$ & $\begin{array}{c}\text { Invierno } \\
27-2-83\end{array}$ \\
\hline Temperatura aire, ${ }^{\circ} \mathrm{C}$ & 22 & 10 \\
\hline Temperatura agua, ${ }^{\circ} \mathrm{C}$ & 21 & 11 \\
\hline $\mathrm{pH}$ & 7,5 & 7,2 \\
\hline Salinidad, g/l & 9 & 7,2 \\
\hline Conductividad, $\mu \mathrm{S} / \mathrm{cm}$ & 13.500 & 9.200 \\
\hline Sólidos en suspensión, mg/1 & 1,27 & 28,65 \\
\hline Alcalinidad, meq/l & 4,14 & 1,69 \\
\hline Cloruros, mg/l & $2.453,4$ & 3.666 \\
\hline Sulfatos, $\mu$ g.atS. $\mathrm{SO}_{4}^{=} / 1$ & 442,86 & 609,5 \\
\hline Nitratos, $\mu$ g.atN. $\mathrm{NO}_{3}^{-} / 1$ & 43,69 & 42,76 \\
\hline Fosfatos, $\mu$ g.atP.PO ${ }_{3}^{3-1} / 1$ & 4,14 & 1 \\
\hline $\mathrm{O}_{2}$ disuelto, $\mathrm{mg} / \mathrm{l}$ & 17 & 6,4 \\
\hline
\end{tabular}

arroyo que drena la cuenca, entre otros, azufre y cloro. Posiblemente el efecto de dilución de las sales disueltas en el agua depende de la naturaleza y composición del sustrato de la cuenca y del estado, fisionomia y cobertura del estrato vegetal. En el caso de la Rambla del Moro, donde los terrenos son muy erosionables y ricos en sales minerales (fundamentalmente cloruros y sulfatos) y el estado de la cubierta vegetal es extraordina- riamente degradado, este efecto es muy patente, hecho que queda de manifiesto en la respuesta negativa de la conductividad al segundo pico de caudal (Figura 3A).

A pesar de ello, los efectos de dilución de sales deben ser compensados por un aumento de la lixiviación y disolución de solutos e iones (entre ellos el calcio) provocada por el agua de escorrentía, aumentando progresivamente la concentra-

Tabla 3.- Valores de los parámetros físicos y químicos medidos en las aguas superficiales de la Rambla del Moro, durante el estudio.

Results of the physico-chernical parameters measured in the Rambla del Moro during the studied period.

\begin{tabular}{|c|c|c|c|c|c|c|c|c|c|c|c|}
\hline $\begin{array}{l}\text { MUESTRAS } \\
\text { Parámetros }\end{array}$ & $\begin{array}{c}1 \\
1-10-86\end{array}$ & $\begin{array}{c}2 \\
10-10-86\end{array}$ & $\begin{array}{c}3 \\
11-10-86 \\
\end{array}$ & $\begin{array}{c}4 \\
12-10-86\end{array}$ & $\begin{array}{c}5 \\
13-10-86 \\
\end{array}$ & $\begin{array}{c}6 \\
14-10-86 \\
\end{array}$ & $\begin{array}{c}7 \\
15-10-86 \\
\end{array}$ & $\begin{array}{c}8 \\
16-10-86 \\
\end{array}$ & $\begin{array}{c}9 \\
17-10-86 \\
\end{array}$ & $\begin{array}{c}10 \\
18-10-86 \\
\end{array}$ & $\begin{array}{c}11 \\
3-11-86 \\
\end{array}$ \\
\hline $\begin{array}{l}\text { Velocidad } \\
\text { corriente, } \mathrm{cm} / \mathrm{s} \\
\text { Caudal }\end{array}$ & 20 & 50 & 33 & 40 & 75 & 50 & 50 & 66 & 66 & 66 & 33 \\
\hline $\begin{array}{c}1 / \mathrm{s} \\
\mathrm{T}^{\mathrm{a}} \text { Agua }\end{array}$ & 32 & 75 & 52,8 & 60 & 396 & 165 & 110 & 180 & 432 & 420 & 51 \\
\hline${ }^{\circ} \mathrm{C}$ & 24 & 22 & 21 & 22 & 21 & 24 & 21 & 20,5 & 18 & 19,5 & 16 \\
\hline $\mathrm{pH}$ & 7,7 & 7,9 & 7,8 & 7,8 & 6,3 & 7,82 & 7,95 & 7,93 & 7,98 & 7,71 & 7,88 \\
\hline & 2,8 & 3 & 3 & 4 & 2,5 & 3,3 & 3 & 3,2 & 1,4 & 1,2 & 5 \\
\hline $\begin{array}{c}\text { Conductividad } \\
\mu \mathrm{S} / \mathrm{cm} \\
\text { Sólidos suspensión }\end{array}$ & 4.410 & 4.700 & 4.700 & 6.500 & 3.900 & 4.850 & 5.000 & 5.500 & 1.900 & 1.900 & 7.000 \\
\hline $\begin{array}{c}\text { Sólidos suspensión } \\
\mathrm{mg} / 1\end{array}$ & 35,5 & 73,1 & 29,8 & 17,4 & 311 & 52,7 & 22,2 & 36,1 & 466 & 587 & 77,8 \\
\hline $\begin{array}{c}\text { Alcalinidad } \\
\text { meq/l } \\
\text { Nitratos }\end{array}$ & 1,28 & 1,2 & 1,22 & 1,33 & 1,56 & 1,2 & 1,24 & 1,4 & 2,23 & 2,52 & 1,33 \\
\hline $\begin{array}{l}\mu \mathrm{g} \text {.atN. } \mathrm{NO}_{3}^{-} / 1 \\
\text { Fosfatos }\end{array}$ & 57,3 & 54,7 & 80,3 & 53,4 & 64,5 & 55,7 & 81,8 & 62,7 & 105,9 & 119,2 & 99,6 \\
\hline$\mu \mathrm{g}$.atP. $\mathrm{PO}_{4}^{3-} /$ & 5,24 & 3,93 & 3,93 & 3,49 & 7,64 & 2,18 & 1,74 & 1,31 & 12,7 & 14,83 & 0,76 \\
\hline
\end{tabular}


ción de carbonatos y bicarbonatos. El coeficiente de correlación entre la alcalinidad y el caudal, resulta significativo $(r=0,84 ; \mathrm{p} \leqslant 0,01)$, pero no lo es para el $\mathrm{pH}$, al mismo nivel $(\mathrm{r}=-0,42$, como cabria esperar en aguas de elevada reserva alcalina (MARGALEF, 1983).

Tanto los nitratos como los fosfatos mantienen correlaciones significativas con el caudal $(\mathrm{r}=0,52$ y $\mathrm{r}=0,82 ; \mathrm{p} \leqslant 0,01$ respectivamente). CaPblane \& Tourend (1978) y Sullivan (1983), entre otros, comprueban que en regiones áridas y semiáridas, el agua de escorrentía transporta concentraciones muy elevadas de nitrógeno, después de una tormenta. En la Rambla del Moro, los nitratos son el único parámetro físico-químico que mantiene concentraciones elevadas después de un mes de haberse producido la riada (Figura 3C). VidAL-ABARCA (1985) apunta observaciones similares en distintos ríos de la cuenca del Segura, al cabo de 4 meses de producirse una avenida de agua. Hay que tener en cuenta que uno de los usos más importantes del suelo de la cuenca es el agrícola, y en los campos de cultivo la pérdida de nitratos por lavado del suelo es extraordinariamente alta (BREZONIK, 1972; KhanNA, 1981). Respecto a los fosfatos, muchos autores han explicado este fenómeno bien por los arrastres de materiales de las tierras de cultivo en las cuencas de drenaje (BAKER, 1985), bien por la liberación de fósforo inorgánico soluble en los sedimentos del cauce (GOLTERMAN, 1984). En cualquier caso, y dada su alta correlación con los sólidos en suspensión $(r=0,95 ; p \leqslant 0,01)$, la recuperación progresiva de las concentraciones normales de fosfatos después de la riada debe ser inducida por procesos de retención y liberación de fósforo y por la incorporación de este elemento a los organismos fotosintetizadores. De hecho, a los 26 días de producirse la avenida apareció en la estación de muestreo un importante tapiz ( $80 \%$ de cobertura) de Enteromorpha sp.

Aún cuando los resultados de este estudio parecen indicar que la estabilización físico-química de la rambla se produce aproximadamente al cabo de un mes, depende siempre del tipo de río donde se desarrolle el fenómeno y de la intensi$\mathrm{d}_{\mathrm{a}} \mathrm{d}$ de la avenida de agua. A ello habría que añadir la frecuencia con que se producen las riadas, que debe provocar un desfase en el equilibrio del sistema.

\section{Bibliografía}

Ávila, A., 1984. Composición química de los arroyos del Montseny (Barcelona). Limnetica 1 (1): 128-135.

BAKER, D.B., 1985. Regional water quality impacts of intensive row-crop agriculture: A lake Erie basin case study. J. Soil and Water Consery. 40 (1): 125-132.

Bormann, F.H. \& G.E. Likens, 1979. Pattern and process in forested ecosystems. Springer-Verlag, New York, 253 pp.

BREZONIK, P.L., 1972. Nitrogen: Sources and transformations in natural waters. pp.: 1-50 In: H.E. Allen \& J.R. KRAMER (eds.), Nutrients in natural waters. John Wiley \& Sons. New York.

CapblanQ, J. \& J.N. TourenQ, 1978. Hydrochimie de la riviere Lot. Annls. Limnol., 14 (1-2): 25-37.

Escarré, A., C. Gracia, F. Roda \& J. Terradas. 1984. Ecología del bosque esclerófilo mediterráneo. Investigación y Ciencia, 95: 69-78.

Fischer, S.G. \& W.L. MiNCKLEY, 1978. Chemical characteristics of a desert stream in flash flood. J. Arid Environ. 1: 25-33.

Fischer, S.G., L.J. Gray, N.B. Grimm \& D.E. Busch, 1982. Temporal sucession in a desert stream ecosystern following flash flooding. Ecol. Monog. 52 (1): 93-110.

Goltermann, H.L. et al., 1978. Methods for physical and chemical analysis of fresh waters. IBP Handbook $n^{\circ} 8,2$ nd ed. Oxford Blackwell, $213 \mathrm{pp}$.

GOLTERMAN, H.L. 1984. Sedirnents, modifying and equilibrating factors in the chemistry of freshwaters. Verh. Internat. Verein. Limnol., 22: 23-59.

HyNES, H.B.N., 1970. The ecology of running waters. University Toronto Press, 555 pp.

INSTITUTO DE HIDROLOG1A, 1980. Análisis de aguas continentales. Métodos recomendados por el grupo de trabajo «Normas analíticas de las Aguas». Madrid. $168 \mathrm{pp}$

KHANNA, P.K., 1981. Leaching of nitrogen from terrestrial ecosystems patterns, mechanisms and ecosystem responses. pp.: 343-352. In: F.E. ClARK \& T. RoswAll (eds.). Terrestrial nitrogen cycles. Processes, ecosystem strategies and management impacts. Ecol. Bull. (Stockholm), 33.

LOPEZ BERMúdEZ, F., et al., 1979. Inundaciones catastróficas, precipitaciones torrenciales y erosión en la provincia de Murcia. Papeles del Dpto. Geografia, 8: 49-91.

López Bermúdez, F. \& D. GutiérRez, 1983. Descripción y experiencias de la avenida e inundación de octubre de 1982 en la cuenca del Segura. Estudios Geogr. Física, 170-171: 87-120.

Lull, H.W. \& K.G. ReInHART, 1972. Forest and floods in eastern Unites States. U.S. Forest Ser. Res. Pap. NE-226. 94 PP.

Margalef, R., 1983. Limnología. Ed. Omega. 1010 PP.

M.O.P.U., 1977. El agua en España. Madrid. Direc. General de Obras Hidráulicas. Centro de estudios Hidrográficos. 288 pp.

M.e.P.U., en prensa. Avenidas e inundaciones. Unidades Temáticas Ámbientales.

Puig, M.A., J. Armengol, G. González, J. Peñuelas, S. SABATER \& F. SABATER, 1987. Chemical and biological changes in the Ter River induced by a series of reservoirs. pp. 363-373. In: CraIG, J. (ed.) Regulated Stream: Advances in Ecology. Plenum Press 
Rodier, J., 1931. Análisis de las aguas. Aguas naturales, aguas residuales, agua de mar. Ed. Omega. $1059 \mathrm{pp}$.

SABATER, S., 1981. Distribución espacio-temporal de las poblaciones de algas del arroyo de L'Avencó (Barcelona). Actas $1^{\circ}$ Cong. Limnología: 159-166.

SuÁREZ, M.L., 1986. Estructura y dinámica de la composición físico-química de las aguas superficiales de una cuenca de características semiáridas del Sureste Español (Río Mula: Cuenca del Segura). Tesis Doctoral. Universidad de Murcia.

SulLIVAN, J.F., 1983. Impact of snowmelt-runoff from agricultural watersheds on dissolved oxygen. Journal WPCF. 55 (2): $170-177$
SundBorg, A., 1983. Los problemas de la sedimentación en las cuencas fluviales. La Naturaleza y sus Recursos, 19(2): 10-21.

VidAL-ABARCA, M.R., 1985. Las aguas superficiales de la Cuenca del Río Segura (S.E. de España). Caracterización físico-química en relación al medio físico y humano. Tesis Doctoral. Universidad de Murcia.

Vidal-Abarca, M.R., C. Montes, L. Ramirez-Diaz \& L. SuArez (en prensa). El clima de la Cuenca del Río Segura (S.E. de España): Factores que lo controlan. Anales de Biología (Ambiental). 